\title{
OPEN Independent evaluation of 12 artificial intelligence solutions for the detection of tuberculosis
}

\author{
Andrew J. Codlin ${ }^{1 凶}$, Thang Phuoc Dao ${ }^{2}$, Luan Nguyen Quang Vo ${ }^{1,2}$, Rachel J. Forse ${ }^{1,3}$, \\ Vinh Van Truong ${ }^{4}$, Ha Minh Dang ${ }^{4}$, Lan Huu Nguyen ${ }^{4}$, Hoa Binh Nguyen ${ }^{5}$, \\ Nhung Viet Nguyen ${ }^{5}$, Kristi Sidney-Annerstedt ${ }^{3}$, Bertie Squire ${ }^{6}$, Knut Lönnroth $^{3}$ \& \\ Maxine Caws ${ }^{6,7}$
}

There have been few independent evaluations of computer-aided detection (CAD) software for tuberculosis (TB) screening, despite the rapidly expanding array of available CAD solutions. We developed a test library of chest X-ray (CXR) images which was blindly re-read by two TB clinicians with different levels of experience and then processed by 12 CAD software solutions. Using Xpert MTB/RIF results as the reference standard, we compared the performance characteristics of each CAD software against both an Expert and Intermediate Reader, using cut-off thresholds which were selected to match the sensitivity of each human reader. Six CAD systems performed on par with the Expert Reader (Qure.ai, DeepTek, Delft Imaging, JF Healthcare, OXIPIT, and Lunit) and one additional software (Infervision) performed on par with the Intermediate Reader only. Qure.ai, Delft Imaging and Lunit were the only software to perform significantly better than the Intermediate Reader. The majority of these CAD software showed significantly lower performance among participants with a past history of TB. The radiography equipment used to capture the CXR image was also shown to affect performance for some CAD software. TB program implementers now have a wide selection of quality CAD software solutions to utilize in their CXR screening initiatives.

An estimated 10 million people developed tuberculosis (TB) globally in 2019 , yet just $71 \%$ of these individuals were reported as treated ${ }^{1}$. The persistent gap between TB treatment and incidence is a major barrier to achieving TB elimination ${ }^{2}$. Numerous intensified and active TB case finding approaches have been piloted over the past decade to identify and treat people with TB who are missed by existing health services ${ }^{3}$. Chest X-ray (CXR) is known to be one of the most sensitive screening tools for TB, but its widespread application in high TB burden countries has traditionally been limited, in line with past World Health Organization policy advising against TB case finding by mobile mass radiography ${ }^{4}$. In recent years, there has been a renewed interest in CXR screening for TB due advances in digital radiography equipment and the advent molecular diagnostic assays ${ }^{5}$.

Several large-scale, community-based CXR screening initiatives have been recently implemented in high TB burden countries ${ }^{6-11}$. These programs show that this case finding approach is both feasible to implement in low- and middle-income countries (LMIC) and effective at identifying people with TB, particularly those with subclinical disease who are frequently missed by TB programs or only diagnosed after long delays ${ }^{12}$. However, most LMIC health systems do not have sufficient capacity to systematically implement this approach. A recent survey of National TB Programs (NTPs) and local implementers from 22 high TB burden countries indicated that 59\% were concerned about shortages of qualified radiologists when planning their own CXR screening initiatives ${ }^{13}$. In addition, high levels of inter- and intra-reader variability can make ensuring the quality of CXR image interpretation a real challenge during programmatic screening activities.

Artificial Intelligence (AI) is one of the fastest growing fields of technology ${ }^{14}$, and it is increasingly being applied to diverse challenges in healthcare, including drug discovery ${ }^{15,16}$, healthcare management ${ }^{17}$, and disease diagnosis $^{18}$. AI also has significant potential to improve TB screening ${ }^{19}$. Multiple computer-aided detection (CAD) software solutions have been developed which can systematically assess and interpret CXR images in

${ }^{1}$ Friends for International TB Relief (FIT), Ho Chi Minh City, Viet Nam. ${ }^{2}$ IRD VN, Ho Chi Minh City, Viet Nam. ${ }^{3}$ Department of Global Public Health, WHO Collaboration Centre on Tuberculosis and Social Medicine, Karolinska Institutet, Solna, Sweden. ${ }^{4}$ Pham Ngoc Thach Hospital, Ho Chi Minh City, Viet Nam. ${ }^{5}$ National Lung Hospital, Ha Noi, Viet Nam. ${ }^{6}$ Department of Clinical Sciences, Liverpool School of Tropical Medicine (LSTM), Liverpool, UK. ${ }^{7}$ Birat Nepal Medical Trust, Kathmandu, Nepal. ${ }^{\circledR}$ email: andrew.codlin@tbhelp.org 
the absence of a radiologist. CAD software for TB screening produce a continuous abnormality score which indicates the likelihood that a CXR image contains an abnormality associated with TB. These scores can then be dichotomized at a selected threshold, above which the CXR image is categorized as abnormal and the individual is indicated for further TB evaluations, such as a sputum-based molecular diagnostic test. The AI algorithms in some CAD solutions will automatically select a cut-off threshold for users, and will continuously use follow-on sputum test result data to optimize threshold selection.

The majority of the published literature on CAD software for TB screening has focused on Delft Imaging's CAD4TB (The Netherlands), which was one of the first commercially available CAD solutions ${ }^{8,11,20-23}$. Two systematic reviews, conducted in 2016 and 2019, also primarily included studies evaluating various versions of the CAD4TB software ${ }^{24,25}$. More recent evaluations have included additional CAD software solutions, including Qure.ai's qXR (India), Lunit's INSIGHT CXR (South Korea), JF Healthcare's JF CXR-1 (China) and InferVision's InferRead DR Chest (Japan $)^{26-29}$. These early evaluations suggest that CAD solutions can match the performance of experienced human readers for detecting abnormalities associated with TB. However, there have been limited reports of independent evaluations applying the technology under programmatic conditions. Continuous software version updates have further complicated the systematic evaluation of different CAD software solutions.

We developed a well-characterized test library of CXR images derived from a community-based, mobile CXR screening initiative in Viet $\mathrm{Nam}^{9}$, and then identified and approached CAD companies for participation in an independent, comparative evaluation of their newest CAD software versions.

\section{Results}

CXR test library characteristics. Of the 1032 participants included in the final test library, 133 (12.9\%) had a positive Xpert result (Table 1$)$. The test library contains more male than female participants $(69.0 \%$ vs $31.0 \%)$ and Xpert positivity is significantly higher in males $(15.0 \%$ vs $8.1 \%, p=0.002)$, consistent with the TB epidemiology in the source population ${ }^{30}$. The test library also contains a higher proportion participants aged $\geq 55$ years $(71.8 \%$ vs $28.2 \%)$, yet Xpert positivity is significantly higher in the younger cohort ( $11.1 \%$ vs $17.5 \%, \mathrm{p}=0.005$ ). Only $39.0 \%$ of test library participants reported having a cough lasting two weeks or longer (a common screening criteria for indicating TB diagnostic evaluations in Viet Nam). $38.2 \%$ of test library participants reported having no cough, fever, weight loss or night sweats. Approximately a third of test library participants (33.5\%) reported having an episode of TB in the past; however, the proportion who were Xpert positive was not significantly different between those with and without a prior episode of TB $(15.0 \%$ vs $12.1 \%, p=0.145)$. Approximately half of the CXR images were captured by each of the library's radiography systems: JPI Healthcare and DRTECH (47.8\% and 52.8\%, respectively). Xpert positivity was significantly higher among participants screened with the DRTECH radiography system (23.9\% vs $6.3 \%, \mathrm{p}<0.001)$. The Expert Reader classified $62.7 \%$ of the images in the test library as Abnormal, while the Intermediate Reader classified $48.0 \%$ of the images as Abnormal. The Intermediate Reader's classifications would have resulted in 24 Xpert positive participants being classified as Normal and not being indicated for further TB testing. The Expert Reader only classified the CXR images of six Xpert positive participants as Normal (4.5\% vs $1.6 \%, \mathrm{p}=0.014)$.

CAD software performance. Table 2 shows the receiver operating characteristic (ROC) area under the curve (AUC) and precision-recall (PR) AUC for each CAD software and Fig. 1 shows their respective ROC curves. Both Qure.ai's qXR v3 and Delft Imaging's CAD4TB v7 achieved a ROC AUC of 0.82, and both software had similar PR AUCs (0.41 for Qure.ai and 0.39 for Delft Imaging). DeepTek's Genki v2 (India) achieved a ROC AUC of 0.78 (0.75-0.82), which is non-significantly lower than the ROC AUC of qXR v3 and CAD4TB v7. Among the software which were evaluated after the provision of outputs by the software developpers, Lunit's INSIGHT CXR v3.1.0.0 was the strongest performer, with a ROC AUC of 0.82 and a PR AUC of 0.44 . The ROC AUC of JF Healthcare's JF CXR-1 v3.0 and InferVision's InferRead DR Chest v1.0.0.0 were non-significantly lower than the ROC AUC of Lunit. The ROC AUC values for the remaining six CAD software ranged from 0.73 to 0.50 .

Comparison of CAD software and human readers. The Expert Reader achieved a sensitivity of 95.5\%, a specificity of $42.2 \%$ and an accuracy of $49.0 \%$ (Table 3 ). When the cut-off threshold for each CAD software was selected to match the $95.5 \%$ sensitivity of the Expert Reader, no CAD software achieved a significantly higher specificity or accuracy. However, Qure.ai's specificity was very close to being significantly higher (Qure.ai: 48.7\% [45.4-52.0\%] vs Expert Reader: 42.2\% [38.9-45.5\%]). Delft Imaging and DeepTek achieved specificity point estimates which were marginally higher than the Expert Reader, while JF Healthcare, OXIPIT and Lunit had specificity point estimates which were marginally lower than the Expert Reader, but these differences were not significant. The six remaining software in the evaluation had a specificity which was significantly lower than the Expert Reader. Despite achieving a lower ROC AUC than InferVision, the specificity of the OXIPIT software was on par with the Expert Reader due to the distribution of the abnormality scores (visible in steep slope change in the ROC curve, Fig. 1).

The Intermediate Reader achieved a sensitivity of $82.0 \%$, a specificity of $57.1 \%$ and an accuracy of $60.3 \%$ (Table 4). When the cut-off threshold was fixed to match the $82.0 \%$ sensitivity achieved by the Intermediate Reader, Qure.ai, Delft Imaging and Lunit achieved a significantly higher specificity and accuracy. DeepTek and JF Healthcare achieved a specificity point estimate which was marginally higher than the Intermediate Reader, while the specificity of InferVision and OXIPIT was slightly lower than the Intermediate Reader. The five remaining software solutions had a specificity which was significantly lower than the Intermediate Reader. 


\begin{tabular}{|c|c|c|c|c|}
\hline & \multirow[b]{2}{*}{ Total (N, \%) } & \multicolumn{2}{|c|}{ Xpert MTB/RIF results } & \multirow[b]{2}{*}{ P-value f $^{+}$} \\
\hline & & Negative (N, \%) & Positive (N, \%) & \\
\hline All participants & 1032 & $899(87.1 \%)$ & $133(12.9 \%)$ & N/A \\
\hline \multicolumn{5}{|l|}{ Demographic factors } \\
\hline \multicolumn{5}{|l|}{ Gender } \\
\hline Male & $712(69.0 \%)$ & $605(85.0 \%)$ & $107(15.0 \%)$ & \multirow{2}{*}{0.002} \\
\hline Female & $320(31.0 \%)$ & $294(91.9 \%)$ & $26(8.1 \%)$ & \\
\hline Age, median (IQR) & $62(53-70)$ & $62(54-71)$ & $59(48-68)$ & 0.004 \\
\hline $15-54$ years & $291(28.2 \%)$ & $240(82.5 \%)$ & $51(17.5 \%)$ & \multirow{2}{*}{0.005} \\
\hline$\geq 55$ years & $741(71.8 \%)$ & $659(88.9 \%)$ & $82(11.1 \%)$ & \\
\hline Health insurance & $881(85.4 \%)$ & $769(87.3 \%)$ & $112(12.7 \%)$ & 0.686 \\
\hline \multicolumn{5}{|l|}{ Residency status } \\
\hline Long-term resident of HCMC & $896(86.8 \%)$ & $783(87.4 \%)$ & $113(12.6 \%)$ & \multirow{2}{*}{0.497} \\
\hline Recent migrant to HCMC & $136(13.2 \%)$ & $116(85.3 \%)$ & $20(14.7 \%)$ & \\
\hline \multicolumn{5}{|l|}{ Presence of TB symptoms } \\
\hline \multicolumn{5}{|l|}{ Cough $(\mathrm{C})$} \\
\hline No Cough & $455(44.1 \%)$ & $420(92.3 \%)$ & $35(7.7 \%)$ & \multirow{3}{*}{$<0.001$} \\
\hline$<2$ weeks & $175(17.0 \%)$ & $148(84.6 \%)$ & $27(15.4 \%)$ & \\
\hline$\geq 2$ weeks & $402(39.0 \%)$ & $331(82.3 \%)$ & $71(17.7 \%)$ & \\
\hline Fever (F) & $56(5.4 \%)$ & $43(76.8 \%)$ & $13(23.2 \%)$ & 0.018 \\
\hline Weight loss (WL) & $113(10.9 \%)$ & $94(83.2 \%)$ & $19(16.8 \%)$ & 0.187 \\
\hline Night sweats (NS) & $64(6.2 \%)$ & $59(92.2 \%)$ & $5(7.8 \%)$ & 0.211 \\
\hline 4 Symptoms: $\mathrm{C}+\mathrm{F}+\mathrm{WL}+\mathrm{NS}$ & $638(61.8 \%)$ & $534(83.7 \%)$ & $104(16.3 \%)$ & $<0.001$ \\
\hline Chest pain & $229(22.2 \%)$ & $199(86.9 \%)$ & $30(13.1 \%)$ & 0.913 \\
\hline Fatigue & $235(22.8 \%)$ & $189(80.4 \%)$ & $46(19.6 \%)$ & $<0.001$ \\
\hline Any TB symptom & $727(70.4 \%)$ & $614(84.5 \%)$ & $113(15.5 \%)$ & $<0.001$ \\
\hline Cough plus any one symptom & $291(28.2 \%)$ & $238(81.8 \%)$ & $53(18.2 \%)$ & 0.001 \\
\hline Any TB symptom except cough & $441(42.7 \%)$ & $373(84.6 \%)$ & $68(15.4 \%)$ & 0.036 \\
\hline \multicolumn{5}{|l|}{ TB risk factors } \\
\hline Contact of TB patient & $64(6.2 \%)$ & $48(75.0 \%)$ & $16(25.0 \%)$ & 0.003 \\
\hline Past history of TB & $346(33.5 \%)$ & $294(85.0 \%)$ & $52(15.0 \%)$ & 0.145 \\
\hline Diabetes & $103(10.0 \%)$ & $87(84.5 \%)$ & $16(15.5 \%)$ & 0.398 \\
\hline HIV & $2(0.2 \%)$ & $2(100.0 \%)$ & $0(0.0 \%)$ & 0.586 \\
\hline \multicolumn{5}{|l|}{ X-ray factors } \\
\hline \multicolumn{5}{|l|}{ Radiography system } \\
\hline JPI Healthcare & $493(47.8 \%)$ & $464(94.1 \%)$ & $29(6.3 \%)$ & \multirow{2}{*}{$<0.001$} \\
\hline DRTECH & $539(52.2 \%)$ & $435(80.7 \%)$ & $104(23.9 \%)$ & \\
\hline \multicolumn{5}{|l|}{ Abnormal CXR } \\
\hline Expert Reader & $647(62.7 \%)$ & $520(80.4 \%)$ & $127(19.6 \%)$ & \multirow{2}{*}{0.338} \\
\hline Intermediate Reader & $495(48.0 \%)$ & $386(78.0 \%)$ & $109(22.0 \%)$ & \\
\hline \multicolumn{5}{|l|}{ Normal/Clear CXR } \\
\hline Expert Reader & $385(37.3 \%)$ & $379(98.4 \%)$ & $6(1.6 \%)$ & \multirow{2}{*}{0.014} \\
\hline Intermediate Reader & $537(52.0 \%)$ & $513(95.5 \%)$ & $24(4.5 \%)$ & \\
\hline
\end{tabular}

Table 1. Demographic and clinical description of participants included in the test library. Significant values are in bold. ${ }^{\neq}$Chi-squared test.

Factors affecting CAD software performance. Table 5 shows the ROC AUC for the top seven performing CAD software (performance at least on par with the Intermediate Reader) disaggregated by key demographic and clinical factors. No software recorded significant differences in ROC AUC between male and female participants; however, the ROC AUC difference by sex of JF Healthcare $(0.74$ vs $0.82, \mathrm{p}=0.057)$ and DeepTek ( 0.75 vs $0.83, p=0.066)$ approached statistical significance. The Delft Imaging (0.86 vs $0.79, p=0.48)$ and JF Healthcare $(0.82$ vs $0.74, \mathrm{p}=0.044)$ software solutions showed significant differences in ROC AUC between younger (15-54 years) and older ( $\geq 55$ years) participants. All seven of the top performing CAD software solutions showed no significant differences between test library participants with and without TB symptoms. However, all but one software (InferVision) showed highly significant differences in ROC AUC between test library participants who reported a history of $\mathrm{TB}$ and those who did not. The largest differences between these two cohorts were recorded by JF Healthcare $(0.82$ vs $0.66, p<0.001)$ and OXIPIT $(0.79$ vs $0.63, p<0.001)$. Lastly, the ROC AUC for the OXIPIT ( 0.77 vs $0.65, \mathrm{p}=0.006)$ and DeepTek ( 0.81 vs $0.72, \mathrm{p}=0.027)$ software varied signifi- 


\begin{tabular}{|l|l|l|}
\hline Developer (software name ${ }^{\mp}$, version) & ROC AUC (95\% CI) & PR AUC (95\% CI) \\
\hline Abnormality scores obtained by FIT & $0.82(0.79-0.86)$ & $0.41(0.33-0.50)$ \\
\hline Qure.ai (qXR v3) & $0.82(0.78-0.85)$ & $0.39(0.31-0.47)$ \\
\hline Delft Imaging (CAD4TB v7) & $0.78(0.75-0.82)$ & $0.28(0.22-0.34)$ \\
\hline DeepTek (Genki v2) & $0.82(0.79-0.86)$ & $0.44(0.35-0.54)$ \\
\hline Abnormality scores provided by CAD company & $0.77(0.73-0.81)$ & $0.28(0.22-0.35)$ \\
\hline Lunit (INSIGHT CXR v3.1.0.0) & $0.76(0.72-0.80)$ & $0.29(0.22-0.36)$ \\
\hline JF Healthcare (JF CXR-1 v3.0) & $0.73(0.69-0.77)$ & $0.23(0.18-0.28)$ \\
\hline InferVision (InferRead DR Chest v1.0.0.0) & $0.70(0.66-0.74)$ & $0.23(0.17-0.29)$ \\
\hline OXIPIT (ChestEye v1) & $0.66(0.61-0.71)$ & $0.23(0.17-0.28)$ \\
\hline Artelus (T-Xnet v1) & $0.66(0.61-0.71)$ & $0.22(0.17-0.28)$ \\
\hline EPCON (XrayAME v1) & $0.53(0.48-0.58)$ & $0.14(0.10-0.17)$ \\
\hline COTO (v1) & $0.50(0.45-0.55)$ & $0.13(0.10-0.16)$ \\
\hline SemanticMD (v1) & & \\
\hline Dr CADx (v0.1) &
\end{tabular}

Table 2. Area under the ROC and precision-recall (RC) curves for each CAD software. ROC AUC area under the receiver operating characteristic curve, $P R A U C$ area under the precision-recall curve. ${ }^{{ }^{*}}$ Software name omitted if none available.
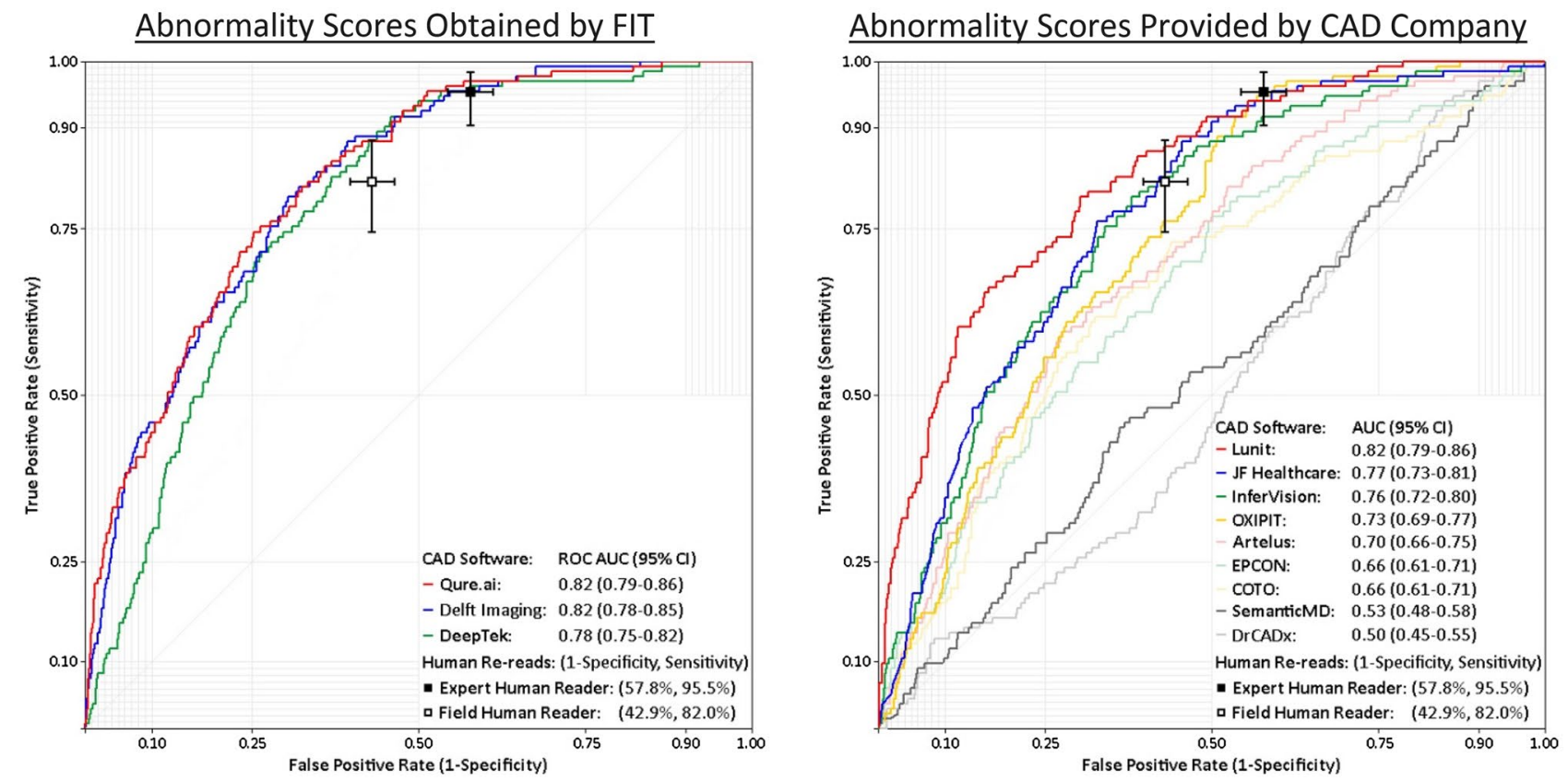

Figure 1. ROC graphs for each CAD software. ROC AUC area under the receiver operating characteristic curve.

cantly depending on the radiography system used to capture the CXR image, while there was weak statistical evidence that the differences observed for the Delft Imaging software were not due to random chance ( 0.82 vs $0.79, \mathrm{p}=0.514)$.

\section{Discussion}

Three CAD software solutions emerged from this evaluation as excellent alternatives for human CXR interpretation, performing on par with the Expert Reader and significantly better than the Intermediate Reader: Qure.ai qXR v3, Delft Imaging CAD4TB v7 and Lunit INSIGHT CXR v3.1.0.0. DeepTek Genki v2 also performed on a par with Expert and Intermediate Readers. Three additional CAD software solutions performed at least on par with the Intermediate Reader.

This evaluation assessed the performance of $12 \mathrm{CAD}$ software solutions for TB screening, which is the largest cross-platform comparative evaluation published to date. This is also the first time six of these CAD solutions have been independently evaluated in the literature. Previous systematic reviews have focused solely on Delft Imaging's CAD4TB ${ }^{24,25}$, and more recent comparative evaluation $s^{26,27,29}$ have included only a limited number of CAD solutions. This independent evaluation highlights the recent significant advances in diagnostic accuracy of 


\begin{tabular}{|c|c|c|c|c|c|c|c|c|}
\hline & Cut-off Score & TP & FP & FN & TN & Sensitivity $(95 \% \mathrm{CI})$ & Specificity $(95 \%$ CI) & Accuracy $(95 \%$ CI) \\
\hline Expert Reader & N/A & 127 & 520 & 6 & 379 & $95.5 \%(90.4-98.3)$ & $42.2 \%(38.9-45.5)$ & $49.0 \%(45.9-52.1)$ \\
\hline \multicolumn{9}{|c|}{ Abnormality scores obtained by FIT } \\
\hline Qure.ai & 44.1 & 127 & 461 & 6 & 438 & $95.5 \%(90.4-98.3)$ & $48.7 \%(45.4-52.0)$ & $54.7 \%(51.7-57.8)$ \\
\hline DeepTek & 31.1 & 127 & 483 & 6 & 416 & $95.5 \%(90.4-98.3)$ & $46.3 \%(43.0-49.6)$ & $52.6 \%(49.5-55.7)$ \\
\hline Delft imaging & 46.7 & 127 & 492 & 6 & 407 & $95.5 \%(90.4-98.3)$ & $45.3 \%(42.0-48.6)$ & $51.7 \%(48.7-54.8)$ \\
\hline \multicolumn{9}{|c|}{ Abnormality scores provided by CAD company } \\
\hline JF Healthcare & 83.4 & 127 & 530 & 6 & 369 & $95.5 \%(90.4-98.3)$ & $41.0 \%(37.8-44.3)$ & $48.1 \%(45.0-51.2)$ \\
\hline OXIPIT & 15.4 & 127 & 532 & 6 & 367 & $95.5 \%(90.4-98.3)$ & $40.8 \%(37.6-44.1)$ & $47.9 \%(44.8-51.0)$ \\
\hline Lunit & 3.0 & 127 & 551 & 6 & 348 & $95.5 \%(90.4-98.3)$ & $38.7 \%(35.5-42.0)$ & $46.0 \%(43.0-49.1)$ \\
\hline InferVision & 53.8 & 127 & 661 & 6 & 238 & $95.5 \%(90.4-98.3)$ & $26.5 \%(23.6-29.5)$ & $35.4 \%(32.5-38.4)$ \\
\hline Artelus & 1.2 & 127 & 691 & 6 & 208 & $95.5 \%(90.4-98.3)$ & $23.1 \%(20.4-26.0)$ & $32.5 \%(29.6-35.4)$ \\
\hline Dr CADx & 27.8 & 127 & 790 & 6 & 109 & $95.5 \%(90.4-98.3)$ & $12.1 \%(10.1-14.4)$ & $22.9 \%(20.3-25.6)$ \\
\hline SemanticMD & 0.4 & 127 & 808 & 6 & 91 & $95.5 \%(90.4-98.3)$ & $10.1 \%(7.2-10.8)$ & $21.1 \%(16.6-21.2)$ \\
\hline EPCON & 0.6 & 127 & 815 & 6 & 84 & $95.5 \%(90.4-98.3)$ & $9.3 \%(6.6-10.0)$ & $20.4 \%(16.0-20.6)$ \\
\hline COTO & 1.5 & 127 & 842 & 6 & 57 & $95.5 \%(90.4-98.3)$ & $6.3 \%(4.8-8.1)$ & $17.8 \%(15.5-20.3)$ \\
\hline
\end{tabular}

Table 3. CAD software performance when matching the sensitivity of the Expert Reader. $T P$ true positive, $F P$ false positive, $F N$ false negative, $T N$ true negative.

\begin{tabular}{|c|c|c|c|c|c|c|c|c|}
\hline & Cut-off Score & TP & FP & FN & TN & Sensitivity $(95 \% \mathrm{CI})$ & Specificity $(95 \% \mathrm{CI})$ & Accuracy (95\% CI) \\
\hline Intermediate Reader & N/A & 109 & 386 & 24 & 513 & $82.0 \%(74.4-88.1)$ & $57.1 \%(53.8-60.3)$ & $60.3 \%(57.2-63.3)$ \\
\hline \multicolumn{9}{|c|}{ Abnormality scores obtained by FIT } \\
\hline Qure.ai & 76.5 & 109 & 307 & 24 & 592 & $82.0 \%(74.4-88.1)$ & $65.9 \%(62.7-69.0)$ & $67.9 \%(65.0-70.8)$ \\
\hline Delft Imaging & 64.7 & 109 & 309 & 24 & 590 & $82.0 \%(74.4-88.1)$ & $65.6 \%(62.4-68.7)$ & $67.7 \%(64.8-70.6)$ \\
\hline DeepTek & 55.7 & 109 & 331 & 24 & 568 & $82.0 \%(74.4-88.1)$ & $63.2 \%(59.9-66.3)$ & $65.6 \%(62.6-68.5)$ \\
\hline \multicolumn{9}{|c|}{ Abnormality scores provided by CAD company } \\
\hline Lunit & 20.7 & 109 & 314 & 24 & 585 & $82.0 \%(74.4-88.1)$ & $65.1 \%(61.9-68.2)$ & $67.2 \%(64.3-70.1)$ \\
\hline JF Healthcare & 98.3 & 109 & 379 & 24 & 520 & $82.0 \%(74.4-88.1)$ & $57.8 \%(54.5-61.1)$ & $60.9 \%(57.9-63.9)$ \\
\hline InferVision & 77.4 & 109 & 387 & 24 & 512 & $82.0 \%(74.4-88.1)$ & $57.0 \%(53.6-60.2)$ & $60.2 \%(57.1-63.2)$ \\
\hline OXIPIT & 23.8 & 109 & 441 & 24 & 458 & $82.0 \%(74.4-88.1)$ & $50.9 \%(47.6-54.3)$ & $54.9 \%(51.9-58.0)$ \\
\hline Artelus & 5.6 & 109 & 492 & 24 & 431 & $82.0 \%(74.4-88.1)$ & $45.3 \%(42.0-48.6)$ & $50.0 \%(46.9-53.1)$ \\
\hline EPCON & 11.7 & 109 & 547 & 24 & 352 & $82.0 \%(74.4-88.1)$ & $39.2 \%(36.0-42.4)$ & $44.7 \%(41.6-47.8)$ \\
\hline COTO & 12.2 & 109 & 568 & 24 & 331 & $82.0 \%(74.4-88.1)$ & $36.8 \%(33.7-40.1)$ & $42.6 \%(39.6-45.7)$ \\
\hline Dr CADx & 64.1 & 108 & 713 & 25 & 186 & $81.2 \%(73.5-87.5)^{\neq}$ & $20.7 \%(18.1-23.5)$ & $28.5 \%(25.8-31.4)$ \\
\hline SemanticMD & 0.9 & 109 & 714 & 24 & 185 & $82.0 \%(74.4-88.1)$ & $20.6 \%(18.0-23.4)$ & $28.5 \%(25.8-31.4)$ \\
\hline
\end{tabular}

Table 4. CAD software performance when matching the sensitivity of the Intermediate Reader. TP true positive, FP false positive, FN false negative; TN True Negative. Bolded figures indicate performance significantly higher than the Intermediate Reader. ${ }^{{ }_{\text {It }}}$ was impossible to select a cut-off score achieving 109 true positives, as two Xpert-positive participants have the same score.

multiple CAD software platforms and also identifies important limitations of the CAD software, which should be addressed in future implementation research.

All seven of these top performing CAD software solutions showed equivalent performance among participants with and without TB symptoms. This finding has important implications for the potential of CAD technology to increase the effectiveness of TB screening programs in identifying people with TB, because approximately half of people with active TB disease in the community do not report having TB symptoms: $30-60 \%$ of people with TB in Africa ${ }^{31}$ and $40-79 \%$ of people with TB in Asia $^{32}$. These individuals can often only be detected through CXR screening, either through community-based screening initiatives or supported by other community referral programs which succeed in overcoming access barriers for facility-based X-ray services ${ }^{33,34}$. CAD software solutions have the potential to reduce CXR access barriers related to shortages of radiologists, particularly those with specialist training in TB.

However, there are key factors which may significantly impair the performance of CAD solutions. Specifically, all but one of the seven top performing CAD software solutions (InferVision) had a significantly lower ROC AUC in people with a history of TB. Participants who had TB in the past may have abnormalities on their CXR images (e.g. fibrotic scarring, nodules without calcification, etc.) which are not indicative of current TB disease. 


\begin{tabular}{|c|c|c|c|c|c|c|c|c|}
\hline & \multicolumn{2}{|l|}{ Qure.ai } & \multicolumn{2}{|l|}{ Delft Imaging } & \multicolumn{2}{|l|}{ DeepTek } & & \\
\hline & $\begin{array}{l}\text { ROC AUC (95\% } \\
\text { CI) }\end{array}$ & P-value $\neq$ & $\begin{array}{l}\text { ROC AUC (95\% } \\
\text { CI) }\end{array}$ & P-value ${ }^{\neq}$ & $\begin{array}{l}\text { ROC AUC (95\% } \\
\text { CI) }\end{array}$ & P-value $\neq$ & & \\
\hline & $0.82(0.79-0.85)$ & - & $0.82(0.78-0.85)$ & - & $0.78(0.74-0.82)$ & - & & \\
\hline \multicolumn{7}{|l|}{ Gender } & & \\
\hline Male & $0.80(0.76-0.85)$ & \multirow{2}{*}{0.222} & $0.80(0.76-0.85)$ & \multirow{2}{*}{0.363} & $0.75(0.71-0.80)$ & \multirow{2}{*}{0.066} & & \\
\hline Female & $0.85(0.79-0.92)$ & & $0.84(0.78-0.90)$ & & $0.83(0.76-0.90)$ & & & \\
\hline \multicolumn{7}{|l|}{ Age group } & & \\
\hline $15-54$ years & $0.84(0.79-0.90)$ & \multirow{2}{*}{0.248} & $0.86(0.81-0.91)$ & \multirow{2}{*}{0.048} & $0.79(0.73-0.85)$ & \multirow{2}{*}{0.534} & & \\
\hline$\geq 55$ years & $0.80(0.76-0.85)$ & & $0.79(0.74-0.84)$ & & $0.77(0.72-0.82)$ & & & \\
\hline \multicolumn{7}{|l|}{$\mathrm{C}+\mathrm{F}+\mathrm{WL}+\mathrm{NS}$} & & \\
\hline No & $0.78(0.71-0.86)$ & \multirow{2}{*}{0.294} & $0.78(0.72-0.85)$ & \multirow{2}{*}{0.307} & $0.75(0.67-0.83)$ & \multirow{2}{*}{0.489} & & \\
\hline Yes & $0.83(0.79-0.87)$ & & $0.82(0.78-0.86)$ & & $0.78(0.74-0.82)$ & & & \\
\hline \multicolumn{7}{|l|}{ History of TB } & & \\
\hline No & $0.86(0.83-0.90)$ & \multirow{2}{*}{0.002} & $0.85(0.82-0.89)$ & \multirow{2}{*}{0.019} & $0.83(0.79-0.87)$ & \multirow{2}{*}{0.001} & & \\
\hline Yes & $0.73(0.65-0.80)$ & & $0.76(0.69-0.83)$ & & $0.69(0.62-0.77)$ & & & \\
\hline \multicolumn{7}{|c|}{ Radiography system } & & \\
\hline JPI Healthcare & $0.85(0.79-0.90)$ & \multirow{2}{*}{0.072} & $0.82(0.76-0.87)$ & \multirow{2}{*}{0.514} & $0.81(0.75-0.86)$ & \multirow{2}{*}{0.027} & & \\
\hline \multirow[t]{4}{*}{ DRTECH } & $0.78(0.73-0.83)$ & & $0.79(0.75-0.84)$ & & $0.72(0.67-0.77)$ & & & \\
\hline & \multicolumn{2}{|l|}{ Lunit } & \multicolumn{2}{|l|}{ JF Healthcare } & \multicolumn{2}{|l|}{ InferVision } & OXIPIT & \\
\hline & $\begin{array}{l}\text { ROC AUC }(95 \% \\
\text { CI) }\end{array}$ & P-value $\neq$ & $\begin{array}{l}\text { ROC AUC (95\% } \\
\text { CI) }\end{array}$ & P-value $\neq$ & $\begin{array}{l}\text { ROC AUC (95\% } \\
\text { CI) }\end{array}$ & P-Value $\neq$ & $\begin{array}{l}\text { ROC AUC }(95 \% \\
\text { CI) }\end{array}$ & P-value ${ }^{+}$ \\
\hline & $0.82(0.79-0.86)$ & - & $0.77(0.73-0.81)$ & - & $0.76(0.72-0.80)$ & - & $0.73(0.69-0.77)$ & - \\
\hline Gender & & & & & & & & \\
\hline Male & $0.81(0.77-0.86)$ & 0373 & $0.74(0.69-0.79)$ & 0057 & $0.75(0.70-0.79)$ & 0712 & $0.70(0.66-0.75)$ & 0718 \\
\hline Female & $0.85(0.79-0.90)$ & $0.5 \%$ & $0.82(0.75-0.90)$ & 0.057 & $0.77(0.68-0.86)$ & 0.112 & $0.77(0.69-0.84)$ & 0.110 \\
\hline Age group & & & & & & & & \\
\hline $15-54$ years & $0.83(0.77-0.89)$ & 0771 & $0.82(0.76-0.87)$ & 0044 & $0.79(0.73-0.85)$ & 0127 & $0.75(0.69-0.81)$ & 0335 \\
\hline$\geq 55$ years & $0.82(0.77-0.86)$ & $0.1 / 1$ & $0.74(0.69-0.79)$ & 0.044 & $0.73(0.67-0.78)$ & 0.127 & $0.71(0.66-0.76)$ & 0.335 \\
\hline $\mathrm{C}+\mathrm{F}+\mathrm{WL}+\mathrm{NS}$ & & & & & & & & \\
\hline No & $0.79(0.72-0.85)$ & 0244 & $0.71(0.63-0.80)$ & 0142 & $0.73(0.64-0.82)$ & 0.525 & $0.72(0.64-0.80)$ & 0991 \\
\hline Yes & $0.83(0.79-0.88)$ & 0.244 & $0.78(0.74-0.83)$ & 0.142 & \begin{tabular}{|l|}
$0.76(0.72-0.81)$ \\
\end{tabular} & 0.525 & $0.72(0.68-0.77)$ & 0.911 \\
\hline History of TB & & & & & & & & \\
\hline No & $0.86(0.82-0.90)$ & 0019 & $0.82(0.77-0.86)$ & 0.001 & $0.78(0.73-0.83)$ & 2120 & $0.79(0.74-0.83)$ & $<0001$ \\
\hline Yes & $0.75(0.68-0.83)$ & 0.019 & $0.66(0.59-0.74)$ & 0.001 & $0.71(0.64-0.78)$ & 0.122 & $0.63(0.55-0.70)$ & $<0.001$ \\
\hline Radiography sy & em & & & & & & & \\
\hline JPI Healthcare & $0.79(0.71-0.86)$ & 0790 & $0.76(0.69-0.83)$ & 0791 & $0.78(0.70-0.86)$ & 0118 & $0.77(0.71-0.84)$ & \\
\hline DRTECH & $0.80(0.75-0.84)$ & 0.190 & $0.75(0.70-0.80)$ & 0.191 & $0.70(0.65-0.75)$ & 0.118 & $0.65(0.60-0.71)$ & 0.0 \\
\hline
\end{tabular}

Table 5. Comparison of CAD software ROC AUC by key demographic and clinical factors. ROC AUC area under the receiver operating characteristic curve. Significant values are in bold. ${ }^{*}$ Chi-squared test.

In these instances, a high CAD software abnormality score may be paired with a negative Xpert test, resulting in diminished software performance. In addition, Xpert testing among people with a history of TB can produce false positive Xpert results many months after a patient has successfully completed treatment ${ }^{35}$. Implementers should be aware of this common limitation when integrating CAD software into their TB programs. CXR images from people with a past history of TB may need an alternative threshold or to be reviewed by an experienced human reader. Software companies should develop, evaluate and refine alternative algorithms for this patient group to optimize software performance.

Although all of the seven top performing CAD software solutions indicated they were radiography system agnostic, we observed a significant impairment in the performance of two solutions (OXIPIT and DeepTek) and possibly a third (Delft Imaging) depending on the radiography system used for CXR image capture. However, the test library evaluated contains only two types of radiography systems, and therefore our data suggests broader independent evaluation of all software solutions against a range of radiography equipment is necessary. Many health systems in high TB burden countries have older and poorly maintained radiography equipment in current use.

The high level of inter-reader variability of CXR images has been well documented in TB programs since the late $1960 \mathrm{~s}^{36}$, particularly among less experienced readers ${ }^{37}$. A strength of this CAD software evaluation was the involvement of two TB clinicians with different levels of experience as benchmarks for the software solutions. This particularly pertains to the inclusion of the Intermediate Reader, as many CAD software evaluations have used a single highly skilled radiologist to re-read the CXR images, thereby setting a very high standard for CAD 
software diagnostic accuracy ${ }^{23,27,29}$. However, experienced expert TB clinicians and radiologists are unlikely to participate in programmatic CXR screening initiatives on a regular basis. The Expert Reader achieved a 95.5\% sensitivity, compared to an $82.0 \%$ sensitivity for the Intermediate Reader. The level of experience of this evaluation's Intermediate Reader is more representative of the field radiologists which Friends for International TB Relief (FIT) employs during mobile CXR screening initiatives. However, the Intermediate Reader is a staff member of a tertiary respiratory hospital, and may be more experienced than generalist radiologist or TB clinicians working at lower-volume secondary and primary care facilities. It is therefore possible that many of the software evaluated in this study would exceed the performance of standard programmatic screening staff, and further evaluations should determine the potential gains in accuracy of screening programs applying CAD solutions.

Now that several CAD software have achieved accuracy exceeding that of human readers, it is also essential to conduct cost effectiveness studies. Our literature review did not find fixed price points published for the CAD software solutions included in this evaluation. Informal feedback from early CAD software adopters has indicated that a unit cost model for each processed DICOM file is commonplace. However, CAD developers may orient themselves on other viable, commonly observed pricing models for SaaS (Software-as-a-Service) solutions, such as per-user subscriptions or price segmentation by time, feature or disease ${ }^{38,39}$. Hybrid pricing models, such as freemium or free/ad-supported solutions, are additional marketing options CAD software developers could consider in light of the increasingly competitive environment of this rapidly expanding market. Lastly, structuring and presenting the chosen pricing model as either value-based or cost-based pricing may also be critical in markets where high-quality and relatively low-cost radiologists are readily available.

Justifying the costs of CAD solutions will most certainly depend on the added value for each individual use case. The FIT mobile CXR screening initiative mobilizes and processes 300 participants per day on average ${ }^{9}$, and one radiologist interprets all of the CXR images in real-time as they are captured throughout the day. In such a high volume setting, CXR interpretation quality and reader fatigue are real concerns ${ }^{40}$. CAD software could be integrated into a screening initiative as an external quality assessment (EQA) tool to identify CXR abnormalities which were missed by the radiologist, or excessive over-reading. Alternatively, the CAD software could be used as a triage tool to identify the totally normal/clear CXR images, reducing the workload of the radiologists and allowing them to prioritize time for reading CXR images which have a higher likelihood of being abnormal. $\mathrm{CAD}$ solutions are currently being integrated into mammography screening programs in high-income countries in a similar fashion ${ }^{41,42}$. Further studies evaluating the implementation experiences, software usability and performance of CAD software solutions in these two contexts are urgently needed, particularly for software where diagnostic performance is already well established.

Our study has several limitations. The test library used in this evaluation contains CXR images collected in one region only. CAD software performance may differ across settings and even between the key populations being screened within a setting. The test library was retrospectively constituted using data from the FIT programmatic mobile CXR screening initiatives, and thus it is biased towards persons with suspected TB. It is likely that the CAD software solutions and human readers would correctly identify true negative CXR images with high accuracy. If this cohort of participants was better represented in the test library, the ROC AUC scores for each CAD software and the specificity for human readers and dichotomized CAD software scores would likely be higher. To overcome this limitation, we identified cut-off thresholds that allowed for a direct comparison of CAD software solutions with human readers, who faced the same challenges associated with the test library's sampling method. We then calculated and compared specificity for the human readers and dichotomized CAD software outputs using Xpert test results as the reference standard for both (primary outcome metric) to minimize the influence of a sampling bias.

A second limitation is that the FIT mobile CXR screening program primarily collected single, spot sputum specimens from participants for Xpert testing. Systematic reviews indicate that the Xpert test has a $99 \%$ sensitivity among smear-positive individuals and an $88 \%$ sensitivity among smear-negative individuals ${ }^{43}$. However, some systematic TB screening initiatives which have used culture as the gold standard have documented Xpert sensitivity as low as $57 \%{ }^{44}$. These data indicate that some test library participants likely have a false negative Xpert result, potentially underestimating CAD software performance. Future CAD evaluations should aim to use the higher sensitivity Xpert MTB/RIF Ultra assay and/or a composite reference standard which includes clinically diagnosed TB after an Xpert-negative result. We were unable to use a composite reference standard in this test library because not all eligible participants underwent a systematic clinical evaluation due to the event-based nature of these campaigns. This evaluation mitigated the impact of unquantified under-diagnosis of TB by focusing on the comparison between human readers and dichotomized CAD software outputs as the primary outcome metric, where performance of both human readers and CAD software were equally affected by the under-diagnosis of TB.

This evaluation collected CAD software outputs using two methods: direct collection by FIT staff who had access to online or box versions of the CAD software and receipt of CAD software outputs from software developers. It is possible that the CAD software developers who received DICOM files from FIT had their own radiologists rapidly grade the test library so they could use their radiologist's interpretations to influence or adjust their CAD software outputs before providing them to FIT. However, this likelihood was deemed to be low, particularly for commercially available CAD solutions, and recent CAD software evaluations have used similar methods for data collection ${ }^{27,29}$. To highlight the differences in data collection methods, and higher levels of trust in the CAD software outputs directly collected by FIT, all analyses in this manuscript have been presented by data collection method.

Despite these limitations, this independent evaluation has conclusively shown that TB program implementers now have a wide, and expanding, selection of accurate CAD software platforms to choose from when designing their programs. Comprehensive prospective operational evaluations are urgently needed to understand the optimal placement of CAD software in TB screening programs. Achieving the potential of CAD software to improve 


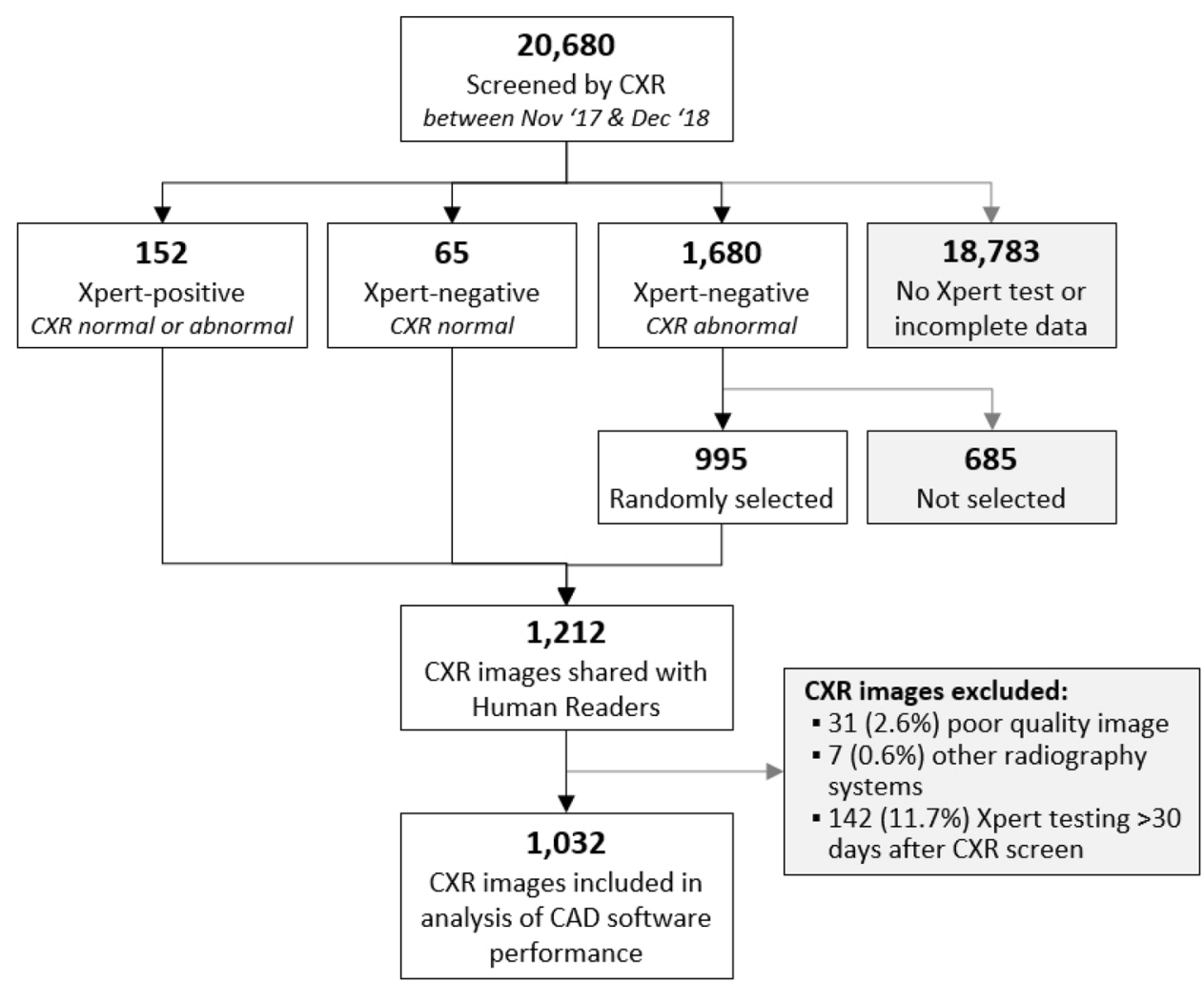

Figure 2. Diagram of test library creation.

TB detection and treatment rates will depend on the availability of investments to scale-up implementation and ensuring optimal value and placement within the TB diagnosis and care cascade.

\section{Methods}

Mobile CXR screening. FIT has been organizing mobile CXR screening events for TB in Ho Chi Minh City, Viet Nam since $2017^{9,33}$. Community members who are at higher risk for TB (e.g. TB contacts, the elderly, people who have TB symptoms, people living far from health facilities, etc.) are mobilized in collaboration with district and commune health authorities and local partners ${ }^{45}$. Participants are first screened for TB symptoms (e.g. cough, fever, night sweats, weight loss, etc.) and other risk factors (history of TB, diabetes, HIV) using a questionnaire on a custom-built mHealth application. They are then referred for CXR screening regardless of their symptom presentation. CXR images are immediately read by a field radiologist and graded as normal or abnormal using the principle of 'intentional over-reading' in line with TB prevalence survey methods ${ }^{46}$. Individuals with an abnormal CXR result are asked to provide a good-quality sputum specimen at the screening event. At the end of each day, specimens are transported to a government laboratory for testing with the Xpert MTB/RIF assay (Cepheid, United States of America [USA]).

Test library creation. DICOM files and clinical data from the mobile CXR screening events organized between 01 November 2017 and 31 December 2018 were used to create a purposively sampled test library for use in CAD software evaluations. The CXR images from 20,680 participants screened during this time period were reviewed for inclusion in the test library (Fig. 2); participants who did not have a valid Xpert test result (mostly because of an initial normal CXR result from the field radiologist), those who were aged less than 15 years, and/ or individuals with foreign objects (e.g. pacemakers, jewelry, underwire, etc.) obscuring their lung fields were excluded. Three types of participants were ultimately selected: (1) all participants $(n=152)$ with a positive Xpert result regardless of their CXR result from the field radiologist, $(2)$ all participants $(n=65)$ with a valid Xpert result after a normal CXR result from the field radiologist (off-algorithm testing), and (3) a randomly selected sample of $60 \%$ of the participants $(n=995)$ with negative Xpert results after an abnormal CXR result from the field radiologist. A test library of 1212 DICOM files was constituted using these initial inclusion criteria. The participant's meta-data inside the DICOM files (e.g. name, birth year and age) were then anonymized.

The test library was sent to two TB clinicians who regularly read CXR images for their respective facilities, for blinded re-reading; the only participant information available to the re-readers was study ID. All CXR images were graded using a standardized interpretation definitions ${ }^{46}$. The Expert Reader had over 30 years of experience working at the Provincial Lung Hospital in Ho Chi Minh City, whereas the Intermediate Reader had 5 years of 
experience working at the Provincial Lung Hospital in Quang Nam, a lower TB burden province in the center of Viet Nam.

The test library was further refined after the blinded re-reads were obtained. Thirty-one CXR images which were graded as poor quality by either the Expert or Intermediate Readers were excluded. A total of seven different radiography systems were used during FIT's mobile CXR screening events; however, just two radiography systems were used for $99 \%$ of the CXR screens. Thus, we excluded the seven CXR images which were captured by the other five radiography systems. Finally, 142 participants who were tested on Xpert more than 30 days after their CXR screen were also excluded. The final test library contains 1032 well-characterized CXR images (Fig. 2).

CAD processing. Sixteen companies offering CAD software for TB screening were identified after a review of the literature and searches on the internet (Artelus, USA; Delft Imaging, The Netherlands; COTO, USA; DeepTek, India; Dr CADx, Zimbabwe; EPCON, Belgium; InferVision, Japan; JF Healthcare, China; JLK, South Korea; Lunit, South Korea; OXIPIT, Lithuania; Quibim, Spain; Qure.ai, India; RadiSen, South Korea; SemanticMD, USA; and Zebra Medical Vision, Israel). 14 companies signed collaboration agreements with FIT which outlined data sharing and the scope of the evaluation (all but Quibim and Zebra Medical Vision). Two companies later withdrew (JLK and RadiSen), leaving 12 companies included in the final evaluation report. Five of the CAD solutions included in this evaluation (DeepTek, CAD4TB, Lunit, Oxipit and Qure.ai) have obtained CE certification to date $\mathrm{e}^{47}$.

DeepTek, Delft Imaging and Qure.ai provided FIT with direct access to their software through either an online user interface or an offline box system. The test library was processed and software outputs were collected directly by FIT staff for these three CAD companies. The test library was shared with all remaining CAD companies via a download link. Staff at these companies processed the DICOM files and provided their software's outputs to FIT within 1 week of data sharing. De-identified demographic and clinical data, including CXR re-reads and Xpert results, were shared with all 12 CAD companies after their software outputs were obtained so these data could be used to train their software algorithms.

Statistical analyses. Descriptive statistics summarizing participant demographics and clinical data were prepared, stratified by Xpert test result, and chi-squared tests were used to measure differences in Xpert positivity. The human reader CXR interpretations were recoded into a binary abnormal/normal result. Abnormal CXR images contained opacities/cavitation/lesions which were possibly caused by TB. CXR images containing abnormalities which the human readers were certain were of non-tubercular origin (e.g., canon ball metastases, vascular abnormalities, emphysema, etc.) were grouped with normal CXR images in this recorded variable. The analysis of CAD software outputs was disaggregated into two groups: abnormality scores obtained directly by FIT and scored provided by the CAD software developers. We first assessed the performance of each CAD software using their continuous abnormality score output. Receiver operating characteristic (ROC) curves were plotted using Xpert test results as the reference standard and areas under the curve (ROC AUCs) were calculated. In addition, we calculated the area under the precision-recall curve (PR AUC), due to the test library's low overall Xpert positivity rate ${ }^{48}$. We then identified two cut-off thresholds to transform the continuous abnormality score of each CAD software into dichotomous normal/abnormal interpretations that matched the sensitivity achieved by the Expert and Intermediate Readers. Performance characteristics of each CAD software were then calculated at these two cut-off thresholds to allow for direct comparisons with human readers (primary outcome metric). For the seven CAD software solutions which performed at least on par with the Intermediate Reader, we calculated and quantitatively compared ROC AUCs ${ }^{49}$ across key demographic and clinical factors, including gender, age group, symptom status, history of TB and radiography system. Statistical analyses were performed using Stata version 13 (StataCorp, USA) and graphs were generated using R version 4.0.0 (R Foundation for Statistical Computing, Austria).

Ethical considerations. Ethical approvals were granted by the Pham Ngoc Thach Hospital Institutional Review Board (430/HDDD-PNT) and the Liverpool School of Tropical Medicine Research Ethics Committee (17-019). Study implementation was approved by the Ho Chi Minh City People's Committee (214/QD-UBND, 2138/QD-UBND). All participants provided written informed consent, and all methods were carried out in accordance with relevant guidelines and regulations. No patient-identifiable data were shared with CAD companies or were used for statistical analyses.

Received: 29 July 2021; Accepted: 30 November 2021

Published online: 13 December 2021

\section{References}

1. World Health Organization. Global Tuberculosis Report 2020 (World Health Organization, 2020).

2. World Health Organization. The End TB Strategy (World Health Organization, 2015).

3. Creswell, J. et al. A multi-site evaluation of innovative approaches to increase tuberculosis case notification: Summary results. PLoS ONE 9(4), e94465 (2014).

4. Miller, C., Lonnroth, K., Sotgiu, G. \& Migliori, G. B. The long and winding road of chest radiography for tuberculosis detection. Eur. Respir. J. 49(5), 1700364 (2017).

5. World Health Organization. WHO Consolidated Guidelines on Tuberculosis Module 2: Screening-Systematic Screening for Tuberculosis Disease (World Health Organization, 2021).

6. Morishita, F. et al. Bringing state-of-the-art diagnostics to vulnerable populations: The use of a mobile screening unit in active case finding for tuberculosis in Palawan, the Philippines. PLoS ONE 12(2), e0171310 (2017). 
7. Camelique, O. et al. Mobile community-based active case-finding for tuberculosis among older populations in rural Cambodia. Int. J. Tuberc. Lung Dis. 23(10), 1107-1114 (2019).

8. Madhani, F. et al. Automated chest radiography and mass systematic screening for tuberculosis. Int. J. Tuberc. Lung Dis. 24(7), 665-673 (2020).

9. Nguyen, L. H. et al. An evaluation of programmatic community-based chest X-ray screening for tuberculosis in Ho Chi Minh City. Vietnam. Trop. Med. Infect. Dis. 5(4), 185 (2020).

10. Codlin, A. J. et al. Results from a roving, active case finding initiative to improve tuberculosis detection among older people in rural Cambodia using the Xpert MTB/RIF assay and chest X-ray. J. Clin. Tuberc. Other Mycobact Dis. 13, 22-27 (2018).

11. Fehr, J. et al. Computer-aided interpretation of chest radiography to detect TB in rural South Africa. MedRxiv. https://doi.org/10. 1101/2020.09.04.20188045 (2020).

12. Frascella, B. et al. Subclinical tuberculosis disease-A review and analysis of prevalence surveys to inform definitions, burden, associations and screening methodology. Clin. Infect. Dis. https://doi.org/10.1093/cid/ciaal402 (2020).

13. Pande, T., Pai, M., Khan, F. A. \& Denkinger, C. M. Use of chest radiography in the 22 highest tuberculosis burden countries. Eur. Respir. J. 46(6), 1816-1819 (2015).

14. Dwivedi, Y. K. et al. Artificial Intelligence (AI): Multidisciplinary perspectives on emerging challenges, opportunities, and agenda for research, practice and policy. Int. J. Inf. Manage. 57, 101994 (2021).

15. Liang, G., Fan, W., Luo, H. \& Zhu, X. The emerging roles of artificial intelligence in cancer drug development and precision therapy. Biomed. Pharmacother. 128, 110255 (2020).

16. Paul, D. et al. Artificial intelligence in drug discovery and development. Drug Discov. Today. 26(1), 80-93 (2021).

17. Mohapatra, S. \& Swarnkar, T. Artificial intelligence for smart healthcare management: Brief study. In Intelligent and Cloud Computing (eds Mishra, D. et al.) 365-373 (Springer, 2021).

18. Shen, J. et al. Artificial intelligence versus clinicians in disease diagnosis: Systematic review. JMIR Med. Inform. 7(3), e10010 (2019).

19. Kulkarni, S. \& Jha, S. Artificial intelligence, radiology, and tuberculosis: A review. Acad. Radiol. 27(1), 71-75 (2020).

20. Maduskar, P. et al. Detection of tuberculosis using digital chest radiography: Automated reading vs interpretation by clinical officers. Int. J. Tuberc. Lung Dis. 17(12), 1613-1620 (2013).

21. Breuninger, M. et al. Diagnostic accuracy of computer-aided detection of pulmonary tuberculosis in chest radiographs: A validation study from sub-Saharan Africa. PLoS ONE 9(9), e106381 (2014).

22. Philipsen, R. H. H. M. et al. Automated chest-radiography as a triage for Xpert testing in resource-constrained settings: A prospective study of diagnostic accuracy and costs. Sci. Rep. 5, 12215 (2015).

23. Rahman, M. T. et al. An evaluation of automated chest radiography reading software for tuberculosis screening among public- and private-sector patients. Eur. Respir. J. 49(5), 1602159 (2017).

24. Harris, M. et al. A systematic review of the diagnostic accuracy of artificial intelligence-based computer programs to analyze chest $\mathrm{x}$-rays for pulmonary tuberculosis. PLoS ONE 14(9), e0221339 (2019).

25. Pande, T., Cohen, C., Pai, M. \& Ahmad, K. F. Computer-aided detection of pulmonary tuberculosis on digital chest radiographs: A systematic review. Int. J. Tuberc. Lung Dis. 20(9), 1226-1230 (2016).

26. Khan, F. A. et al. Chest X-ray analysis with deep learning-based software as a triage test for pulmonary tuberculosis: A prospective study of diagnostic accuracy for culture-confirmed disease. Lancet Digit. Health 2(11), e573-e581 (2020).

27. Qin, Z. Z. et al. Using artificial intelligence to read chest radiographs for tuberculosis detection: A multi-site evaluation of the diagnostic accuracy of three deep learning systems. Sci. Rep. 9(1), 1-10 (2019).

28. Nash, M. et al. Deep learning, computer-aided radiography reading for tuberculosis: A diagnostic accuracy study from a tertiary hospital in India. Sci. Rep. 10(1), 1-10 (2020).

29. Qin, Z. Z. et al. Tuberculosis detection from chest $\mathrm{x}$-rays for triaging in a high tuberculosis-burden setting: An evaluation of five artificial intelligence algorithms. Lancet Digit Health. 3(9), e543-e554 (2021).

30. Nguyen, H. V. et al. The second national tuberculosis prevalence survey in Vietnam. PLoS ONE 15(4), e0232142 (2020).

31. Law, I. \& Floyd, K. National tuberculosis prevalence surveys in Africa, 2008-2016: An overview of results and lessons learned. Trop. Med. Int. Health 25(11), 1308-1327 (2020).

32. Onozaki, I. et al. National tuberculosis prevalence surveys in Asia, 1990-2012: An overview of results and lessons learned. Trop Med Int Health 20(9), 1128-1145 (2015).

33. Vo, L. N. Q. et al. A comparative impact evaluation of two human resource models for community-based active tuberculosis case finding in Ho Chi Minh City, Viet Nam. BMC Public Health 20(1), 934 (2020).

34. Mac, T. H. et al. Optimizing active tuberculosis case finding: Evaluating the impact of community referral for chest X-ray screening and Xpert testing on case notifications in two cities in Viet Nam. Trop. Med. Infect. Dis. 5(4), 181 (2020).

35. Theron, G. et al. False-positive Xpert MTB/RIF results in retested patients with previous tuberculosis: Frequency, profile, and prospective clinical outcomes. J. Clin. Microbiol. https://doi.org/10.1128/JCM.01696-17 (2018).

36. Nyboe, J. Results of the international study on X-ray classification. Bull. Int. Union Tuberc. 41, 115-124 (1968).

37. Kosack, C. S. et al. Evaluation of a chest radiograph reading and recording system for tuberculosis in a HIV-positive cohort. Clin. Radiol. 72(6), e1-e9 (2017).

38. Abdat, N., Spruit, M. \& Bos, M. Software as a service and the pricing strategy for vendors. In Digital Product Management, Technology and Practice Interdisciplinary Perspectives, 154-192 (2010).

39. Ferrante, D. Software licensing models: What's out there? IT Prof. 8(6), 24-29 (2006).

40. Stec, N., Arje, D., Moody, A. R., Krupinski, E. A. \& Tyrrell, P. N. A systematic review of fatigue in radiology: Is it a problem? Am. J. Roentgenol. 210(4), 799-806 (2018).

41. Dembrower, K. et al. Effect of artificial intelligence-based triaging of breast cancer screening mammograms on cancer detection and radiologist workload: A retrospective simulation study. Lancet Digit. Health 2(9), e468-e474 (2020).

42. McKinney, S. M. et al. International evaluation of an AI system for breast cancer screening. Nature 577(7788), 89-94 (2020).

43. Horne, D. J. et al. Xpert MTB/RIF and Xpert MTB/RIF Ultra for pulmonary tuberculosis and rifampicin resistance in adults. Cochrane Database Syst. Rev. 6, CD009593 (2019).

44. Sander, M. et al. Systematic screening for tuberculosis among hospital outpatients in Cameroon: The role of screening and testing algorithms to improve case detection. J. Clin. Tuberc. Other Mycobact. Dis. 15, 100095 (2019).

45. Vo, L. N. Q. et al. Evaluating the yield of systematic screening for tuberculosis among three priority groups in Ho Chi Minh City, Viet Nam. Infect. Dis. Poverty 9(1), 166 (2020).

46. World Health Organization. Tuberculosis Prevalence Surveys: A Handbook (World Health Organization, 2011).

47. FIND. Digital Chest Radiography and Computer-Aided Detection (CAD) Solutions for Tuberculosis Diagnosis: Technology landscape analysis (FIND, 2021).

48. Cook, J. \& Ramadas, V. When to consult precision-recall curves. Stand. Genomic Sci. 20(1), 131-148 (2020).

49. Cleves, M. A. From the help desk: Comparing areas under receiver operating characteristic curves from two or more probit or logit models. Stand. Genomic Sci. 2(3), 301-313 (2002). 


\section{Acknowledgements}

The authors would like to thank staff at the National Lung Hospital, the People's Committee and Department of Health for Ho Chi Minh City, Pham Ngoc Thach Hospital, the District TB Units of District 06, 08, 12, Binh Chanh, Go Vap, Hoc Mon and Tan Bin and the Ho Chi Minh City Public Health Association for their support during implementation of mobile CXR screening events in 2017 and 2018 . We would also like to thank Dr. Nguyen Duc Bang and Dr. Bui Ngoc Minh for blindly re-reading the test library's CXR images.

\section{Author contributions}

A.J.C., L.N.Q.V. and R.J.F. conceived and designed the evaluation. A.J.C., T.D., L.N.Q.V., R.J.F., V.V.T., H.M.D. and L.H.N. supervised program implementation and data collection. A.J.C. and T.D. compiled programmatic data for this evaluation, which were analyzed by A.J.C. A.J.C. wrote the first draft of the manuscript. A.J.C., T.D., L.N.Q.V., R.J.F., V.V.T., H.M.D., L.H.N., H.B.N., N.V.N., K.S.A., B.S., M.C. and K.L. reviewed the manuscript and interpreted data.

\section{Funding}

This evaluation was supported by the European Commission Horizon 2020 IMPACT TB Grant 733174. Additional support was provided by the Stop TB Partnership's TB REACH initiative, with funding from Global Affairs Canada. These funding agencies had no role in the design of the study, in collection, analysis, and interpretation of data, or in writing the manuscript.

\section{Competing interests}

The authors declare no competing interests.

\section{Additional information}

Correspondence and requests for materials should be addressed to A.J.C.

Reprints and permissions information is available at www.nature.com/reprints.

Publisher's note Springer Nature remains neutral with regard to jurisdictional claims in published maps and institutional affiliations.

(c) (i) Open Access This article is licensed under a Creative Commons Attribution 4.0 International License, which permits use, sharing, adaptation, distribution and reproduction in any medium or format, as long as you give appropriate credit to the original author(s) and the source, provide a link to the Creative Commons licence, and indicate if changes were made. The images or other third party material in this article are included in the article's Creative Commons licence, unless indicated otherwise in a credit line to the material. If material is not included in the article's Creative Commons licence and your intended use is not permitted by statutory regulation or exceeds the permitted use, you will need to obtain permission directly from the copyright holder. To view a copy of this licence, visit http://creativecommons.org/licenses/by/4.0/.

(C) The Author(s) 2021 